\title{
LIFE EXTINCTIONS BY COSMIC RAY JETS
}

\author{
Arnon Dar, Ari Laor, and Nir J. Shaviv \\ Department of Physics and Space Research Institute \\ Israel Institute of Technology, Haifa 32000, Israel.
}

\begin{abstract}
High energy cosmic ray jets from nearby mergers or accretion induced collapse (AIC) of neutron stars (NS) that hit the atmosphere can produce lethal fluxes of atmospheric muons at ground level, underground and underwater, destroy the ozone layer and radioactivate the environment. They could have caused most of the massive life extinctions on planet Earth in the past $600 M y$. Biological mutations due to ionizing radiations could have caused the fast appearance of new species after the massive extinctions. An early warning of future extinctions due to NS mergers may be obtained by identifying, mapping and timing all the nearby binary neutron stars systems. A warning of an approaching cosmic ray burst from a nearby NS merger/AIC may be provided by a very intense gamma ray burst which preceeds it.
\end{abstract}

\section{Introduction}

The early history of single-celled organisms during the Precambrian, 4560 to 570 million years $(M y)$ ago, is poorly known. Since the end of the Precambrian the diversity of both marine and continental life increased exponentially. Analysis of fossil record of microbes, algae, fungi, protists, plants and animals shows that this diversification was interrupted by five major mass extinction "events" and some smaller extinction peaks [1] . The "big five" mass extinctions occurred in the Late Ordovician, Late Devonian Late Permian, Late Triassic and end-Cretaceous and included both marine and continental life. The largest extinction occurred about $251 M y$ ago at the end of the Permian period. The global species extinction ranged then between $80 \%$ to $95 \%$, much more than, for instance, the end-Ordovician extinction $439 M y$ ago which eliminated $57 \%$ of marine genera, or the Cretaceous-Tertiary extinction $64 M y$ ago which killed the dinosaurs and claimed $47 \%$ of existing genera [2]. In spite of intensive studies it is still not known what caused the mass extinctions, how quick were they and whether they were subject to regional variations. Many extinction mechanisms have been proposed but no single mechanism seems to provide a satisfactory explanation of both the marine and continental extinction levels, the biological extinction patterns and the repetition rate of the mass extinctions $[1,2]$. These include astrophysical extinction mechanisms, such as meteoritic impact that explains the iridiun anomaly which was found at the Cretaceous/Tertiary boundary [3] but has not been found in all the other extinctions [4], supernova 
explosions [5] and gamma ray bursts [6], which do not occur close enough at sufficiently high rate to explain the observed rate of mass extinctions.

In this paper we propose that high energy cosmic ray jets (CRJs) from mergers or accretion induced collapse (AIC) of neutron stars (NS) that hit the atmosphere of an Earth-like planet can produce lethal fluxes of atmospheric muons at ground level, underground and underwater, destroy the ozone layer and radioactivate the environment. Nearby NS mergers/AIC can explain the massive extinction on the ground, underground and underwater and the higher survival levels of radiation resistant species and terrain sheltered species in the five "great" extinctions in the past $600 \mathrm{My}$. More distant galactic mergers/AIC can cause smaller extinctions. Biological mutations due to ionizing radiations may explain the fast appearance of new species after massive extinctions. Intense cosmic ray bursts enrich rock layers with detectable traces of cosmogenically produced radioactive nucleides such as ${ }^{129} \mathrm{I},{ }^{146} \mathrm{Sm},{ }^{205} \mathrm{~Pb}$ and ${ }^{244} \mathrm{Pu}$. Tracks of high energy particles in rock layers on Earth and on the moon may also contain records of intense cosmic irradiations. An early warning of future extinctions due to neutron star mergers can be obtained by identifying, mapping and timing all the nearby binary neutron stars systems. A final warning of an approaching CRJ from a nearby neutron stars merger is provided by a very intense gamma ray burst a few days before the arrival of the CRJ.

\section{Cosmic Ray Jets From NS Mergers}

Three NS-NS binaries are presently known within the galactic disc; B1913+16 [7] at a distance of $D \sim 7.3 \mathrm{kpc}, \mathrm{B} 203+46[8]$ at $D \sim 2.3 \mathrm{kpc}$, and B1534+12 [9] at $D \sim 0.5 \mathrm{kpc}$, and B2127+11C [10] in the globular cluster M15 at $D \sim 10.6 \mathrm{kpc}$. Although unseen as radio pulsars, the companion stars in these systems have been identified as neutron stars from their mass which is inferred via measurements of the relativistic periastron advance using standard pulse timing techniques [11]. The continuous energy loss through gravitational radiation brings the two NS closer and closer until they merge. It is believed that their final merger releases an enormous amount of gravitational binding energy, $\sim M_{\odot} c^{2}$, in a few ms, in the form of gravitational waves, neutrinos and kinetic energy of relativistic ejecta. It was suggested that the neutrinos and/or the relativistic ejecta from NS merger/AIC in distant galaxies produce the mysterious gamma ray bursts (GRBs), that occur at a rate of about one per day, although the mechanism which converts their energy into gamma rays is still not clear [12].

It is also believed that due to strong gravitational tidal forces the final NS-NS or NS-BH merger proceeds through the formation of an accretion disc. Observations seem to indicate that highly collimated jets are ejected by all systems where matter is undergoing disc accretion onto a compact central object $[13,14]$. They also indicate that the jet kinetic energy is a considerable fraction of the accretion power and that the jets reach enormous distances without being de- 
flected by galactic or intergalactic magnetic fields: The highly relativistic and highly collimated jets from active galactic nuclei (AGN), which are believed to be powered by mass accretion onto a massive black hole at a typical rate of $\left(\sim M_{\odot} y^{-1}\right)$, reach a distance up to a million light years before disruption [13]. Highly collimated relativistic matter that is ejected sporadicly by microquasars (superluminal galactic sources such as GRS 1915+105 [14, 15], GRO J165-40 [16]) and by X-ray sources such as SS 433 [17] and Cygnus X-3 [18], which are are close binary systems where mass is accreted onto a neutron star or a stellar black hole from a companion star, seem to reach hundreds of light years before disruption. The exact mechanism by which the gravitational and electromagnetic fields around these accreting and rotating compact objects produce the highly relativistic jets is still unknown. However, the instantaneous accretion rates $\left(\gg M_{\odot} s^{-1}\right)$ and the strength of the magnetic fields which are involved in the final stage of merger/AIC of compact stellar objects in compact binary systems are probably many orders of magnitude larger than those encountered in AGN. Therefore, it is natural to expect that highly relativistic jets are also ejected in mergers/AIC of compact stellar objects, are highly collimated and reach hundreds of light years, or more, before disruption. These jets may produce the cosmological gamma ray bursts by internal radiation and/or interaction with the external medium. After disruption they are isotropized by the galactic magnetic field and form the galactic cosmic rays. If they hit an Earth-like planet before disruption, they can devastate all forms of life on it.

\section{Constraints From GRBs and Cosmic Rays}

Jets ejected by NS mergers/AIC in distant galaxies may explain cosmological GRBs [19]. In view of the uncertainties in modeling jet ejection in NS merger/AIC, rather than relying on numerical simulations we inferred [19] from the observed properties of GRBs that the ejected jets have typical Lorentz factors of $\Gamma \sim 10^{3}$, beaming angles $\Delta \Omega \leq 1 / 100$ similar to those observed/estimated for AGN and microquasars, and ejected mass $\Delta M \sim(d M / d \Omega) \Delta \Omega \leq 10^{-4} M_{\odot}$ (i.e., released kinetic energy bounded by $E_{K}=\Gamma \Delta M c^{2}<0.1 M_{\odot} c^{2} \sim 2 \times$ $10^{53} \mathrm{erg}$ ).

The finite life times of close binaries due to gravitational radiation emission have been estimated [12] from the observed binary period, orbital eccentricity and the masses of the pulsar and its companion. They have been used to estimate that the NS-NS merger rate in the Milky Way (MW) disc is [20] $R_{M W} \sim$ $10^{-4}-10^{-5} y^{-1}$. Beamed ejection from NS mergers/AIC requires merger/AIC rate of compact objects in the entire Universe (at GRBs redshifts) of the order of $10^{5}-10^{6} y^{-1}$. It is larger by $1 / \Delta \Omega \sim 10^{2}$ than that required by spherical explosions. It is, however, in good agreement with the updated observational and theoretical estimates of the merger rates of compact objects in the Milky Way, which yield values in the range $10^{5}-10^{6} y^{-1}$ mergers per Universe [21], 
instead of initial estimates [20] of $\sim 10^{3}-10^{4} y^{-1}$. Thus, the updated estimates of the NS-NS merger rate in the Universe are consistent with the observed rate [12] of GRBs (approximately one per day) and with the injection rate of cosmic rays in the MW that is required in order to maintain a constant cosmic ray flux in the MW:

The escape rate of cosmic rays from the MW requires an average injection rate of $Q_{C R} \sim 10^{41} \mathrm{erg} \mathrm{s}^{-1}$, in high energy cosmic rays above $\mathrm{GeV}$, in order to maintain a constant energy density of cosmic rays in the MW [22]. This injection rate can be supplied by NS-NS mergers if the injected jet energy per merger is $E_{K} \sim 10^{53} \mathrm{erg}$ and if the NS-NS merger rate in the disc of the MW is $R_{M W} \sim 10^{-4}-10^{-5} y^{-1}$ :

$$
Q_{C R}^{N S} \sim R_{M W} E_{K} \sim 3 \times 10^{40}-3 \times 10^{41} \operatorname{erg~s}^{-1}
$$

\section{Attenuation of CRJs}

The highly relativistic jets from quasars and microquasars do not seem to be attenuated efficiently in the interstellar or the intergalactic space. This is unlike the non relativistic ejecta in supernova ( $\mathrm{SN}$ ) explosions, which is attenuated by Coulomb collisions in the interstellar medium (ISM) over a distance of a few $p c$ : Moderately energetic charged particles, other than electrons, lose energy in neutral interstellar gas primarily by ionization. The mean rate of energy loss (or stopping power) is given by the Bethe-Bloch formula

$$
-d E / d x \approx\left(4 \pi Z^{2} n_{e} \beta^{-2} e^{4} / m_{e} c^{2}\right)\left[(1 / 2) \ln \left(2 m_{e} c^{2} \beta^{2} \Gamma^{2} T_{\max } / I^{2}\right)-\beta^{2}\right]
$$

where $Z e$ is the charge of the energetic particle of mass $M_{i}$, velocity $\beta c$ and total energy $E=\Gamma M_{i} c^{2}, n_{e}$ is the number of electrons per unit volume in the medium in atoms with ionization potential $I$, and $T_{\max }=2 m_{e} c^{2} \beta^{2} \Gamma^{2} /\left(1+2 \gamma m_{e} / M_{i}\right)$. If the interstellar gas around the SN is ionized (by the initial UV flash), then $I$ has to be replaced by $e^{2} / R_{D}$ where $R_{D}=\left(k T / 4 \pi e^{2} n_{e}(Z+1)\right)^{1 / 2}$ is the Debye screening length. Thus, for SN ejecta with $Z \sim 1$ and $v \sim 10000 \mathrm{~km} \mathrm{~s}^{-1}$ in an ionized interstellar medium with a typical density of $n_{H} \sim 1 \mathrm{~cm}^{-3}$, and $k T \sim 1 \mathrm{eV}$ the stopping distance of the ejecta due to Coulomb interactions is $x \sim E / 2(d E / d x) \sim 6 p c$. In fact, the stopping of the ejecta by Coulomb interactions is consistent with observations of SN remnants, like SN 1006 [23], while the assumption that the ionized interstellar medium is glued to the swept up magnetic field seems to be contradicted by some recent observations [24]. The range increases with energy like $\beta^{4}$ until nuclear collisions become the dominant loss mechanism. The range of nuclei with $\Gamma \sim 1000$ in a typical interstellar density of $n_{H} \sim 1 \mathrm{~cm}^{-3}$, is approximately $\sim 10^{25} \mathrm{~cm}$, i.e., much larger than galactic distances.

Although the galactic magnetic field, $H \sim 3-5 \times 10^{-6}$ Gauss, results in a Larmor radius of $r_{L}=\beta \Gamma m c / q H \sim 10^{15} \mathrm{~cm}$ for protons with $\Gamma=1000$, it 
does not deflect and disperse significantly jets from NS-NS mergers at distances smaller than $\sim 1 k p c$ from the explosion. That can be concluded from the fact that accretion jets from forming stars, microquasars and AGN reach distances of tens, hundreds, and million light years, respectively, without significant deflection or attenuation. Probably, because of their high particle and energy densities the jets produce internal magnetic fields which shield them from the interstellar magnetic field and allow them to follow almost free balistic trajectories in the interstellar medium.

\section{$5 \quad$ Mass Extinctions By CRJs}

We assume that the ambient interstellar gas is not swept up with the jet. If it were, then the jet would not reach even $\sim 10 \mathrm{pc}$. In SN explosions collective modes are invoked as the source of the coupling of the ejecta to the interstellar medium, required in order to attenuate the SN debris. As mentioned above, binary Coulomb interactions are sufficient to produce the observed coupling in SN explosions, and coupling through collective modes is not necessarily present. Due to internal magnetic fields the jets are highly collimated, not deflected and probably reach distances of $D \sim 1 k p c$, where the internal energy density becomes compareble to the external (magnetic and radiation) energy density.

Unattenuated jets from NS-NS mergers can be devastating to life on nearby planets: At a distance of $1 k p c$ their duration is

$$
\delta t \sim D / 2 c \Gamma^{2} \sim 1 \text { day }-2 \text { months }
$$

for typical values of $\Gamma$ between 1000 and 100, respectively. The time integrated energy flux of the jet at $D \sim 1 \mathrm{kpc}$ is, typically, $\sim 10^{12} \mathrm{TeV} \mathrm{cm}{ }^{-2}$. Thus, the energy deposition in the atmosphere by the jet is equivalent to the total energy deposition of galactic cosmic rays in the atmosphere over $\sim 10^{7} y$. However, the typical energy of the cosmic rays in the CRJ is $\sim 1 \mathrm{TeV}$ per nucleon, compared with $\sim 1 \mathrm{GeV}$ per nucleon for ordinary cosmic ray nuclei. Collisions of such particles in the atmosphere generate atmospheric cascades where a significant fraction of the CRJ energy is converted into "atmospheric muons" through leptonic decay modes of the produced mesons. Most of these muons do not decay in the atmosphere because of their high energy, unlike most of the atmospheric muons which are produced by ordinary cosmic rays. The average number of high energy muons produced by nucleons of primary energy $E_{p}$, which do not decay in the atmosphere and reach sea level with energy $>E_{\mu}$ at zenith angle $\theta<\pi / 2$, is given approximately by [25]:

$$
<N_{\mu}>\sim\left(0.0145 E_{p}[\mathrm{TeV}]\right)\left(E_{p} / E_{\mu}\right)^{0.757}\left(1-E_{\mu} / E_{p}\right)^{5.25} / \cos \theta .
$$

Thus a jet with energy of about $1 \mathrm{TeV}$ per nucleon at a distance of $1 \mathrm{kpc}$ produces at sea level a flux of atmospheric muons of

$$
I_{\mu}(>3 \mathrm{GeV}) \sim 10^{12} \mathrm{~cm}^{-2} .
$$


Such muons deposit energy in matter via ionization. Their energy deposition rate is [26] $-d E / d x \geq 2 \mathrm{MeV} g^{-1} \mathrm{~cm}^{-1}$. The whole-body lethal dose from penetrating ionizing radiation resulting in $50 \%$ mortality of human beings in 30 days [26] is $\leq 300 \mathrm{rad} \sim 2 \times 10^{10} /(d E / d x) \sim 10^{10} \mathrm{~cm}^{-2}$ where $d E / d x$ is in rate is in $M e V g^{-1} \mathrm{~cm}^{-1}$ units. The lethal dosages for other vertebrates can be a few times larger while for insects they can be as much as a factor 20 larger. Hence, a CRJ at $D \sim 1 k p c$ which is not significantly dispersed by the galactic magnetic field produces a highly lethal burst of atmospheric muons. Because of muon penetration, the large muon flux is lethal for most species even deep (hundreds of meters) underwater and underground, if the cosmic rays arrive from well above the horizon. Thus, unlike the other suggested extraterrestrial extinction mechanisms, a CRJ which produces a lethal burst of atmospheric muons can explain also the massive extinction deep underwater and why extinction is higher in shallow waters.

Although half of the planet is in the shade of the CRJ, planet rotation exposes a larger fraction of the planet surface to the CRJ. Additional effects increase the lethality of the CRJ over the whole planet. They include:

(a) The pollution of the environment by radioactive nuclei, produced by spallation of atmospheric and surface nuclei by shower particles. Using the analytical methods of [27], we estimate that for an Earth-like atmosphere, the flux of energetic nucleons which reaches the surface is also considerable,

$$
I_{p}(>100 \mathrm{MeV}) \sim I_{n}(>100 \mathrm{MeV}) \sim 10^{10} \mathrm{~cm}^{-2} .
$$

Global winds spread radioactive gases in a relatively short time over the whole planet.

(b) Depletion of stratospheric ozone by the reaction of ozone with nitric oxide, generated by the cosmic ray produced electrons in the atmosphere (massive destruction of stratospheric ozone has been observed during large solar flares which produced energetic protons [28]).

(c) Extensive damage to the food chain by radioactive pollution and massive extinction of vegetation and living organisms by ionizing radiations (the lethal radiation dosages for trees and plants are slightly higher than those for animals but still less than the flux given by eq. 5 for all except the most resilient species).

\section{Signatures of CRJ Extinction}

The biological extinction pattern: The biological extinction pattern due to a CRJ depends on the exposure and the vulnerability of the different species to the primary and secondary effects of the CRJ. The exposure of the living organisms to the muon burst depends on the intensity and duration of the CRJ, on its direction relative to the rotation axis of Earth (Earth shadowing), on the local sheltering provided by terrain (canyons, mountain shades) and by underwater and underground habitats, and on the risk sensing/assessment and 
mobility of the various species. The lethality of the CRJ depends as well on the vulnerability of the various living species and vegetation to the primary ionizing radiation, to the drastic changes in the environment (e.g., radioactive pollution and destruction of the ozone layer) and to the massive damage and radioactive poisoning of the food chain. Although the exact biological signature may be quite complicated, and somewhat obscured in fossil records (due to poor or limited sampling, deterioration of the rocks with time and dating and interpretation uncertainties because of bioturbational smearing) it may show the general pattern expected from a CRJ extinction. Indeed, a first examination of the fossil records suggest that there is a clear correlation between the extinction pattern of different species, their vulnerability to ionizing radiation and the sheltering provided by their habitats and the environment they live in. For instance, insects which are less vulnerable to radiation, were extinct only in the greatest extinction - the end-Permian extinction $251 M y$ ago. Even then only 8 out of 27 orders were extinct compared with a global species extinction that ranged between $80 \%$ to $95 \%$ [4]. Also plants which are less vulnerable to ionizing radiation suffered lower level of extinction. Terrain, underground and underwater sheltering against a complete extinction on land and in deep waters may explain why certain families on land and in deep waters were not extinct even in the great extinctions, while most of the species in shallow waters and on the surface were extinct [4]. Mountain shadowing, canyons, caves, underground habitats, deep underwater habitats and high mobility may also explain why many species like crocodiles, turtles, frogs, (and most freshwater vertebrates), snakes, deep sea organisms and birds were little affected in the Cretaceous/Tertiary $(\mathrm{K} / \mathrm{T})$ boundary extinction which claimed the life of the big dinosaurs and pterosaurs. In particular, fresh underground waters in rivers and lakes are less polluted with radioisotopes and poisons produced by the CRJ than sea waters and may explain the survival of freshwater amphibians.

Geological Signatures: The terrestrial deposition of the primary CRJ nuclides or the production of stable nuclides in the atmosphere or in the surface by the CRJ is too small to be detectable. In particular, the proposed mechanism cannot explain the surface enrichment at the $\mathrm{K} / \mathrm{T}$ boundary by about $3 \times 10^{5}$ tons of iridium. Alvarez et al. [3] suggested that the impact of extraterrestrial asteroid, with an Ir abundance similar to that observed in early solar system Chondritic meteorites whose Ir abundance is larger than crustal Ir abundance by $\sim 10^{4}$, could cause the Ir anomaly and explain the mass extinction at the $\mathrm{K} / \mathrm{T}$ boundary. However, no significant Ir enrichment was found in all other mass extinctions. Moreover, other isotopic anomalies due to meteoritic origin have not been found around the $\mathrm{K} / \mathrm{T}$ boundary; in particular the As/Ir and $\mathrm{Sb} / \mathrm{Ir}$ ratios are three orders of magnitude greater than chondritic values but are in accord with a mantle origin [29]. Extensive iridium measurements showed that the anomaly does not appear as a single spike in the record, indicative of an instantaneous event, but rather occur over a measurable time interval of 10 to $100 \mathrm{ky}$ or possibly longer [30]. That and the high abundance of Ir 
in eruptive magma led to the suggestion that the iridium anomaly is due to a global volcanic activity over 10 to $100 \mathrm{kyr}$ at the K/T boundary [30,31] which also caused the $\mathrm{K} / \mathrm{T}$ extinction: Eruptions have a variety of short term effects, including cooling from both dust and sulfates ejected into the stratosphere, acid rain, wildfires, release of poisonous elements and increase in ultraviolet radiation from ozone-layer depletion. But, examination of major volcanic eruptions in the past $100 \mathrm{My}$ have shown that none of them greatly affected the diversity of regional and global life on land or in the oceans [4].

A CRJ will enhance the abundance of stable cosmogenic isotopes in the geological layer corresponding to the CRJ event, but, the enrichment may be negligible compared to their accumulation through long terrestrial exposure of the geological layers to galactic cosmic rays prior to the CRJ. However, CRJ enrichment of sediments with unstable radioisotopes of mean lifetimes much shorter than the age of the solar system, $\tau \ll t_{\odot} \approx 4570 M y$, but comparable to the extinction times, may be detectable through low traces mass spectrometry. In particular, fission of long lived terrestrial nuclei, such as ${ }^{238} \mathrm{U}$ and ${ }^{232} \mathrm{Th}$, by shower particles, and capture of shower particles by such nuclei, may lead to terrestrial production of, e.g., ${ }^{129} \mathrm{I}$ with $\tau=15 M y,{ }^{146} \mathrm{Sm}$ with $\tau=146 \mathrm{My}$, ${ }^{205} \mathrm{~Pb}$ with $\tau=43 \mathrm{My}$ and ${ }^{244} \mathrm{Pu}$ with $\tau=118 \mathrm{My}$, respectively. These radioisotopes may have been buried in underwater sediments and underground rocks which were protected from further exposure to cosmic rays. The main background to such a CRJ signature is the continuous deposition by cosmic rays and by meteoritic impacts on land and sea. Cosmic rays may include these trace radioisotopes due to nearby sources (e.g., supernova explosions) and because of spallation of stable cosmic ray nuclei in collisions with interstellar gas. Meteorites may include these trace elements due to a long exposure in space to cosmic rays.

Finally, large enhancement of $\mathrm{TeV}$ cosmic ray tracks in magma from volcanic eruptions coincident with extinctions may also provide fingerprints for CRJ extinctions.

\section{Rate of Mass Extinctions}

Assuming that the spatial distribution of NS binaries and NS mergers in the MW follow the distribution of single pulsars [],

$$
d N \propto e^{-R^{2} / 2 R_{0}^{2}} e^{-|z| / h} R d R d z,
$$

with a disc scale length, $R_{0} \sim 4.8 k p c$, and a scale height, $h>0.5 k p c$ perpendicular to the disc and independent of disc position, we find that the average rate of CRJs from NS-NS mergers that reach planet Earth from distances $\leq 1 k p c$, is $\sim 10^{-8} y^{-1}$. It is consistent with the 5 big extinctions which have occurred during the last $600 \mathrm{My}$ in the Paleozoic and Mesozoic eras. The relative strengths 
of these extinctions may reflect mainly different distances from the CRJs. Beyond $\sim 1 k p c$ from the explosion the galactic magnetic field begins to disperse the CRJ and suppresses its lethality. Such CRJs, if not too far, can still cause partial extinctions at a higher rate and induce biological mutations which may lead to the appearance of new species.

The galactic rate of SN explosions is $\sim 100 y^{-1}$. The range of debris from SN explosions in the interstellar medium is shorter than $10 \mathrm{pc}$. The rate of SN explosions within a distance of $10 p c$ from Earth which follows from eq. 7 is $R_{S N}(<10 p c) \approx 10^{-10} y^{-1}$. High energy cosmic rays which, perhaps, are produced in the SN remnant by shock acceleration, carry only a small fraction of the total explosion energy and arrive spread in time due to their diffusive propagation in the interstellar magnetic field. Also neutrino and light emissions similar to those observed in SN1987A, at a distance of a few $p c$ cannot cause a mass extinction.

\section{Conclusions}

Cosmic Ray Bursts from neutron star mergers may have caused the massive continental and marine life extinctions which interrupted the diversification of life on our planet. Their rate is consistent with the observed rate of mass extinctions in the past $570 \mathrm{My}$. They may be able to explain the complicated biological and geographical extinction patterns. Biological mutations induced by the ionizing radiations which are produced by the CRJs may explain the appearance of completely new species after extinctions. A first examination suggests a significant correlation between the biological extinction pattern of different species and their exposure and vulnerability to the ionizing radiation produced by a CRJ. The iridium enrichment around the Cretaceous/Tertiary extinction that claimed the life of the dinosaurs and pterosaurs cannot be due to a CRJ. It may have been caused by intense volcanic eruptions around that extinction. Isotopic anomaly signatures of CRJ extinctions may be present in the geological layers which recorded the extinctions. Elaborate investigations of the effects of CRJs from relatively nearby neutron star mergers and their biological, radiological and geological fingerprints are needed before reaching a firm conclusion whether the massive extinctions during the long history of planet Earth were caused by CRJs from neutron stars mergers. If nearby neutron star mergers are responsible for mass extinctions, then an early warning of future extinctions due to neutron star mergers can be obtained by identifying, mapping and timing all the nearby binary neutron stars systems. A final warning for an approaching CRJ from a nearby neutron-stars merger will be provided few days before its arrival by a gamma ray burst produced by the approaching CRJ.

Acknowledgement: This research was supported in part by the Technion fund for promotion of research.

References 
1. M.J. Benton, Science 278, 52 (1995) and references therein.

2. D.H. Erwin, Scientific American, 275, 72 (1996) and references therein.

3. L.W. Alvarez et al., Science 208, 1095 (1980).

4. D.H. Erwin, Nature, 367, 231 (1994) and references therein.

5. M.A. Ruderman, Science, 184, 1079 (1974); J. Ellis, et al., ApJ. 470, 1227 (1996).

6. S.E. Thorsett, ApJ. 444, L53 (1995).

7. R.A. Hulse \& J. Taylor, ApJ. 195, L51 (1975).

8. G.H. Stokes et al., ApJ. 294, L21 (1991).

9. A. Wolszcan, Nature 350, 688 (1991).

10. S.B. Anderson et al., Nature 346, 42 (1990).

11. R.N. Manchester \& J.H. Taylor, Pulsars, (Freeman, San Francisco 1977);

J.H. Taylor \& J.M. Weisberg, ApJ. 345, 434 (1989).

12. G.J. Fishman \& C.A.A. Meegan, Ann. Rev. Astr. Ap. 33, 415 (1995).

13. See, e.g., P. Padovani, these proceedings and references therein.

14. See, e.g., I.F. Mirabel, these proceedings and references therein.

15. I.F. Mirabel and L.F. Rodriguez, Nature, 371, 46 (1994).

16. S.J. Tingay et al., Nature, 374, 141 (1995).

17. See, e.g., B. Margon, Ann. Rev. Ast. Ap., 22, 57, (1988)

18. R.G. Strom et al., Nature, 337, 234 (1989).

19. N.J. Shaviv, \& A. Dar, Submitted to PRL, astro-ph 9606032.

20. E.S. Phinney, ApJ. 380, L17 (1991); R. Narayan, T. Piran and I. Shemi, ApJ, 379, L17 (1991); S.J. Curran, \& D.L. Lorimer, MNRAS 276, 347 (1995);

See, however, L.V. Tutukov and A.R. Yungelston, MNRAS, 260, 675 (1993); V.M. Lipunov et al., ApJ, 454, 493 (1995).

21. See, e.g., E.P.J. van den Heuvel and D.R. Lorimer, MNRAS 283, L37 (1996); V.M. Lipunov et al., Ap. \& Sp. Phys. Rev. 9, 1 (1996).

22. V.S. Berezinsky et al., Ast. of Cosmic Rays, (North Holland 1990) p. 71.

23. C.C. Wu et al., ApJ. 416, 247 (1993).

24. A. Dar, A. Laor, \& N.J. Shaviv, to be published.

25. J.W. Elbert, Proc. DUMAND Workshop (ed. A. Roberts) 2, p. 101 (1978).

26. R.M. Barnett et al., Phys. Rev. D 54, 1 (1996).

27. A. Dar, Phys. Rev. Lett. 51, 227 (1983).

28. J.A.E. Stephenson et al., Nature 352, 137 (1991).

29. C.B. Officer, \& C.L. Drake, Science, 227, 1161 (1985).

30. See, e.g., C.B. Officer et al., Nature 326, 143 (1987).

31. See, e.g., L. Zhao \& F.T. Kyt, Earth planet Sci. Lett. 90, 411 (1988). 